\title{
Les conventions entre le CNRS et l'université de Strasbourg : une expérience pionnière
}

\section{Françoise Olivier-Utard}

\section{(2) OpenEdition}

\section{Journals}

Édition électronique

URL : https://journals.openedition.org/histoire-cnrs/691

DOI : 10.4000/histoire-cnrs.691

ISSN : 1955-2408

Éditeur

CNRS Éditions

Édition imprimée

Date de publication : 5 novembre 2004

ISBN : 978-2-271-06249-9

ISSN : 1298-9800

\section{Référence électronique}

Françoise Olivier-Utard, «Les conventions entre le CNRS et l'université de Strasbourg : une expérience pionnière », La revue pour l'histoire du CNRS [En ligne], 11 | 2004, mis en ligne le 04 septembre 2007, consulté le 20 mai 2021. URL : http://journals.openedition.org/histoire-cnrs/691 ; DOI : https://doi.org/ 10.4000/histoire-cnrs.691

Ce document a été généré automatiquement le 20 mai 2021.

Comité pour l'histoire du CNRS 


\title{
Les conventions entre le CNRS et l'université de Strasbourg : une expérience pionnière
}

\author{
Françoise Olivier-Utard
}

Introduction

1 L'organisation de recherche en France après la Seconde Guerre mondiale ne se fit pas aisément, mais l'université de Strasbourg fut l'une des premières à surmonter les antagonismes et à contribuer à la mise en place de relations institutionnelles entre les organismes. Elle signa une convention avec le CNRS dès 1952, concernant le Centre de recherches macromoléculaires (CRM), laboratoire propre du CNRS dirigé par Charles Sadron et une seconde en 1956, concernant l'Institut de recherches nucléaires (IRN), laboratoire universitaire dirigé par Serge Gorodetzky. Ainsi, près de quinze ans avant la création des laboratoires associés ou des équipes mixtes, l'université de Strasbourg et le CNRS s'associaient autour de deux centres de recherches emblématiques des nouvelles problématiques scientifiques.

2 On peut s'interroger sur les motivations qui ont présidé à ces rapprochements, car ces deux laboratoires avaient, à leur création, des statuts forts différents : le premier était un laboratoire propre du CNRS, le second un laboratoire spécifiquement universitaire. Ces cheminements convergents soulèvent plusieurs questions. Ces institutions auraient-elles bénéficié d'un contexte particulier à Strasbourg? Ou bien est-ce le champ disciplinaire de la physique, en pleine restructuration en France après la guerre, qui aurait généré cette construction inédite? Quel fut le rôle joué par la personnalité des protagonistes strasbourgeois?

Charles Sadron fut l'un des ténors des débats sur les missions du CNRS et nombre de ceux qui participèrent aux débats, comme Louis Néel, Émile Terroine, Edmond Bauer ou André Lichnerowitz étaient, ou avaient été, en poste à Strasbourg. Quelle fut leur influence? Enfin, quel a été l'impact de cette initiative sur les autres équipes scientifiques? Est-elle passée inaperçue au milieu des grands débats nationaux sur les projets de politique de la recherche? Les documents dont nous disposons sont des 
archives, plus ou moins dispersées ${ }^{1}$, des monographies inédites mais riches $^{2}$, des articles $^{3}$ et des entretiens avec des chercheurs de ces deux instituts ont complété les sources écrites ${ }^{4}$.

La recherche scientifique strasbourgeoise à la Libération

4 Les deux instituts concernés, le CRM et l'IRN, furent créés après la guerre, dans le contexte très particulier de l'histoire de l'Alsace marquée par deux annexions allemandes depuis 1870. La reconstitution de l'université se fit tardivement et par étapes. Rappelons d'abord qu'à la fin de la guerre, l'université de Strasbourg était encore repliée à Clermont-Ferrand, où elle avait été évacuée en 1939 et où elle avait, tant bien que mal, tenté de résister aux agressions allemandes. Les Allemands en effet, après avoir annexé de fait l'Alsace et la Moselle, avaient d'une part ouvert à Strasbourg l'une des quatre universités nazies d'Allemagne (Reichsuniversität) et d'autre part tenté de démanteler l'université repliée en exigeant de récupérer une partie du matériel de laboratoire déménagé à Clermont-Ferrand en 1939 et en opérant plusieurs rafles au cœur des locaux universitaires clermontois. Des étudiants et des professeurs, au total plus d'une centaine de personnes, avaient été arrêtés, internés, et certains d'entre eux, déportés dans les camps de concentration. Les scientifiques avaient été particulièrement visés lors de la grande rafle du 25 novembre 1943. Parmi les savants dont il va être question ici, Charles Sadron et Albert Kirrmann avaient été déportés à Buchenwald, Marc Klein à Auschwitz. À travers les individus, c'est l'université de Strasbourg en tant que telle qui avait été visée. Le traumatisme était profond. Le poème d'Aragon qui évoque cette expérience martyre ${ }^{5}$, et les témoignages des rescapés ${ }^{6}$, publiés en 1947, soulignent l'acharnement nazi à mettre à bas l'université alsacienne.

5 Le calendrier de la Libération a lui aussi son importance. La ville de Strasbourg ne fut libérée que le 23 novembre 1944 et la région en février 1945 seulement. La remise en route de l'université française à Strasbourg ne commença qu'au printemps 1945, avec le retour des hospices civils et de la faculté de médecine et se poursuivit en octobre avec celui de la faculté des sciences. Les déportés survivants des camps furent libérés en mai 1945. La rentrée officielle eut lieu le 23 novembre 1945. À cette date, beaucoup de tractations avaient eu lieu. Bien des places étaient prises, bien des ambitions déçues.

6 L'université de Strasbourg avait ceci de particulier que la recherche y avait été très développée d'abord pendant l'annexion allemande de 1870 à 1918, puis par ceux qui avaient reconstitué l'université française en 1919. Ainsi, par exemple, Pierre Weiss y avait développé une importante école du magnétisme, au sein de laquelle avaient été formés Louis Néel et Charles Sadron. Le premier CNRS avait d'ailleurs contribué au financement d'un puissant électroaimant pour ce laboratoire dès 1937. De même, chez les mathématiciens, des initiatives originales avaient vu le jour à Strasbourg dans l'entre-deux-guerres, comme la naissance du groupe Bourbaki. Pendant la Seconde Guerre mondiale, les Nazis avaient, quant à eux, construit un instrument qui fut fort convoité à la Libération : un accélérateur de particules Cockcroft-Walton de 1,5 millions de volts, implanté dans l'enceinte des hospices civils- faculté de médecine ${ }^{8}$, et mis en service en février 1944 par le physicien Rudolf Fleischmann.

L'IRN, de l'université au CNRS

7 C'est la présence de cet instrument puissant et quasiment unique en Europe qui justifia la création de l'un des deux laboratoires qui nous intéressent ici, l'Institut de recherches nucléaires (IRN). L'existence de cet accélérateur renvoyait à la question que les Alliés se posaient: les Allemands possédaient-ils la puissance atomique? Une 
commission américaine se rendit à Strasbourg pour examiner l'appareil. Elle en conclut que les Allemands n'avaient pas atteint un niveau suffisant pour maîtriser la réaction nucléaire. L'appareil gardait toutefois un intérêt national : c'était l'accélérateur le plus puissant disponible sur le sol français. Frédéric Joliot lui-même n'avait récupéré comme prise de guerre allemande qu'un instrument plus faible. Mais s'il y eut tentative de sa part d'en prendre directement le contrôle, elle échoua.

8 En effet, rentré de Suisse à Strasbourg en février 1945, Lucien Pautrier, le directeur de la clinique dermatologique, sur le terrain de laquelle se trouvait le bâtiment qui abritait l'accélérateur, proposa un marché en juillet de la même année : l'appareil serait livré à qui reconstruirait la clinique dermatologique touchée par les bombardements. Dans son esprit, la remise en service de l'accélérateur devait se faire selon l'objectif allemand initial : produire des éléments radioactifs destinés à la médecine. C'est en effet dans ce but que Rudolf Fleischmann en avait demandé et obtenu la construction. Or, l'appareil, commandé en 1942 mais livré en 1944 seulement, n'avait pratiquement jamais fonctionné, à cause de difficultés techniques rencontrées lors du montage, de pannes d'électricité liées à la guerre, et enfin de bombardements américains dans les alentours immédiats. Et il n'y avait plus personne sur place capable de le remettre en route.

Lucien Pautrier, alors que les physiciens étaient encore retenus à Clermont, s'adressa en avril 1945 à Frédéric Joliot, directeur du CNRS et lui proposa la direction du «laboratoire d'applications biologiques de la physique et de la chimie nucléaire de la faculté de médecine de Strasbourg». Frédéric Joliot proposa les noms de ses collaborateurs proches pour les différents départements envisagés, Charles Leblond, Marguerite Perey et André Berthelot. Charles Leblond fit un rapport qui suggérait une collaboration avec la faculté des sciences. Mais la faculté de médecine fit savoir qu'elle n'entendait pas s'associer à la faculté des sciences pour exploiter un matériel dont elle revendiquait la pleine propriété. Elle tenait à garder la maîtrise de l'instrument en le confiant à Marc Klein, un médecin, de retour d'Auschwitz. Frédéric Joliot récusa cette solution : ce n'était pas à ses yeux l'homme de la situation car il était histologiste et non biologiste. La faculté de médecine tint bon. Frédéric Joliot prit ses distances.

Une situation très confuse s'en suivit, tant au sujet de la propriété qu'au sujet de la direction du futur institut. Le CNRS semble avoir été désigné d'abord comme propriétaire de ce bien allemand mis sous séquestre par le ministère de l'Intérieur mais, avec possibilité pour l'université d'en demander le transfert à l'Éducation nationale, tandis que les Hospices civils considérèrent que tout leur appartenait. En mars 1946, le recteur fut chargé, après une entrevue stérile entre les plus hautes instances du CNRS et celles de l'Éducation nationale, de constituer une commission de liquidation. L'union sacrée se fit alors entre les facultés de médecine, de pharmacie et de sciences. Le doyen de la faculté des sciences, Gabriel Foëx, proposa de recruter le physicien Serge Gorodetzky, qu'il avait connu comme maître de conférences à Clermont-Ferrand, pour lui confier dès que possible la direction d'un institut interfacultaire associant les facultés de médecine, sciences et pharmacie. Créé en juillet 1946, il prit en février 1947 le nom d'Institut de recherches nucléaires (IRN). Ses statuts furent envoyés pour approbation à Paris en mars 1947. Le conseil d'administration comprenait trois membres du CNRS: Frédéric Joliot, Marguerite Perey et Émile Terroine. Le conseil du 23 janvier 1948 nomma le physicien Serge Gorodetzky à la tête du laboratoire. Les fonds débloqués par le CNRS dès 1945 pour la remise en marche de l'appareil furent utilisés. On fit appel à celui qui avait procédé à la première 
installation, l'ingénieur allemand Kuntke. En novembre 1948, l'appareil était prêt à fonctionner. L'IRN existait de fait, malgré le retard prolongé pour la signature de ses statuts. Après d'invraisemblables errances, ces derniers finirent par retrouver le chemin de Strasbourg, le 5 janvier 1951.

L'examen de ces statuts montre qu'il s'agissait bien d'un laboratoire universitaire, avec un budget propre. Mais la représentation du CNRS et du CEA dans le conseil d'administration souligne l'envergure nationale de l'Institut. L'originalité de l'IRN résidait dans son autonomie par rapport au CEA dont Frédéric Joliot venait de prendre la direction, mais cette autonomie n'empêchait pas que des liens étroits entre différentes institutions ne se nouent, à travers le conseil d'administration et des aides matérielles diverses. Serge Gorodetzky sut par la suite jouer habilement des conflits de la guerre froide pour obtenir des financements substantiels.

Quelle était la mission de l'IRN? À l'origine, et comme les statuts l'indiquent, elle se calquait sur celle dont Rudolf Fleischmann avait été investi mais qu'il n'avait pu mettre en œuvre. Il s'agissait d'organiser la recherche dans trois directions: la physique nucléaire, la chimie nucléaire et la biologie. Ces recherches devaient déboucher sur des applications médicales, ce qui explique le choix d'implantation du bâtiment initial dans l'enceinte de l'hôpital. Mais cet objectif, jamais vraiment suivi, fut vite abandonné : Serge Gorodetzky était un physicien, formé par Louis Leprince-Ringuet. Il était intéressé par la recherche pure sur la structure nucléaire. La recherche appliquée ne le tentait pas, et dans tous les cas, il était fort éloigné de toute idée de fabrication de produits radioactifs escomptés par la faculté de médecine, comme la suite le révéla. L'Institut relevait de l'université, et offrait des postes de professeurs, tandis que le CNRS, pour reprendre l'analyse d'Antoine Prost ${ }^{9}$, fonctionna comme une agence de moyens, en y affectant des chercheurs et des techniciens et en finançant du matériel. Il est important de souligner en outre qu'il s'agissait du seul laboratoire de physique nucléaire français qui ne fût pas rattaché au CEA. Le statut de certains personnels CNRS était tout à fait particulier. Il existait en effet, créé par Frédéric Joliot, un corps d'ingénieurs et techniciens spécifique pour les personnels attachés aux accélérateurs, avec des salaires attractifs.

13 L'Institut s'organisa d'abord autour de Serge Gorodetzky, entouré de quatre attachés de recherche (Jean Combes, Jean-Jacques Jung, Théo Muller, Denise Magnac-Valette) et d'un technicien. La politique de recrutement de la faculté des sciences favorisa le laboratoire. On y recruta en mars 1949 Marguerite Perey, pour laquelle une chaire de chimie nucléaire fut créée (la seconde en France après Paris) et, en octobre 1949, Pierre Cüer, maître de conférences. Marguerite Perey donna son cours inaugural en novembre de la même année. Un ingénieur d'aéronautique, collaborateur de Frédéric Joliot au Laboratoire de synthèse atomique, Jacques Thirion, arriva lui aussi en 1949 - tandis qu'André Coche, un collaborateur de Marguerite Perey à l'Institut du radium, arriva en 1950. Le renforcement de l'équipe de chercheurs se fit donc par des créations de postes universitaires pour d'anciens collaborateurs de Frédéric Joliot, et par la promotion de personnels venus d'autres institutions, comme l'Institut du radium. C'était là une possibilité de carrière intéressante dans une période où les chercheurs du CNRS n'avaient pas de statut et où le titre de professeur s'accompagnait en revanche d'un prestige certain.

Assez vite, l'Institut se scinda, pour des raisons qui ne furent pas toujours scientifiques, en plusieurs unités, qui devinrent des laboratoires différents essaimés dans la ville. En 
1951, il y en avait trois : le laboratoire du CNRS de Serge Gorodetzky, qui travaillait avec Pierre Armbruster, André Gallmann et Albert Knipper sur la structure des noyaux, dans l'enceinte de l'hôpital; et deux laboratoires de faculté, le laboratoire de chimie nucléaire de Marguerite Perey, qui travaillait avec André Coche et Pierre Adloff, dans le bâtiment de la rue Goethe ; et le laboratoire de physique corpusculaire de Pierre Cüer, qui travaillait sur les émulsions nucléaires et les radiations ionisantes des plaques photographiques, dans les locaux de l'Institut de physique, rue de l'Université. Cette dernière équipe était composée de 6 chercheurs: Jean Combes, Henri Braun, JeanJacques Jung, Jean-Pierre Longchamp et Denise Magnac-Valette. Ainsi les contacts avec la faculté de médecine étaient-ils rompus dès cette époque. La collaboration interfacultaire annoncée ne se fit pas non plus. Les tentatives de collaboration ultérieures avec des biologistes furent chaotiques.

De l'IRN au CRN

15 L'Institut prit de l'ampleur au fil des ans. Henri Longchambon, venu en 1952 inaugurer les nouveaux locaux du Centre de recherches macromoléculaires, proposa de prendre exemple sur ce centre pour lancer une opération nouvelle, conjointement avec l'université. Le CNRS était de plus en plus sollicité pour financer le fonctionnement de l'Institut et il souhaitait avoir une plus grande responsabilité dans sa gestion. Il signa donc une convention avec l'université en 1956 et proposa de prendre en charge la construction d'un nouvel accélérateur (Van de Graaf, 6 millions de volts), dans de nouveaux locaux. L'IRN prit alors le nom de Centre de recherches nucléaires (CRN) et emménagea dans la banlieue de Strasbourg, à Cronenbourg, au sein d'un grand complexe scientifique. Les différents laboratoires furent rassemblés et le dispositif fut complété par un département d'applications biologiques, confié à Jean-Henri Vivien. Les statuts du nouveau centre de recherches furent rédigés sur le modèle de ceux du CRM présentés plus loin. Le support financier de l'université continua à être très important, en particulier grâce aux collectifs budgétaires qui permettaient une certaine souplesse dans la répartition des crédits.

En 1971, le CRN fut rattaché à l'Institut national de physique nucléaire et de physique des particules (IN2P3) du CNRS, dans le cadre d'une nouvelle politique scientifique nationale de coordination des centres de recherche nucléaire. Le CRN devenait « laboratoire associé » par convention tacitement renouvelée tous les ans.

Le texte fut signé par Jean Teillac, directeur de l'IN2P3, et par Guy Ourisson, président de l'université scientifique nouvellement créée par éclatement de l'ancienne université strasbourgeoise après les événements de mai 1968. Le Centre de recherches nucléaires nouvelle formule se constitua avec un directeur unique, mais il conserva de ses anciennes structures des liens avec l'enseignement et la recherche universitaire. Le président de l'université et un représentant de son conseil scientifique siégeaient statutairement au comité de direction du CRN. Sur le plan pédagogique, l'IN2P3 s'engageait à accueillir des étudiants de troisième cycle de Strasbourg. Sur le plan financier, l'IN2P3 et l'université mettaient à la disposition du CRN des moyens qui devaient être affectés à son seul profit. Le CRN garda donc une certaine autonomie, ou du moins une identité particulière, assortie d'une certaine souplesse dans sa gestion.

L'évolution de l'Institut décrivit donc une trajectoire qui alla de l'université vers le CNRS, dans une collaboration ininterrompue. Il était entendu, dès 1945, que la cause de la physique nucléaire était nationale, mais que la situation particulière laissée à Strasbourg par les Allemands justifiait une structure locale spécifique. La création de 
l'IRN résulte de la conjoncture strasbourgeoise. À l'origine du laboratoire, il y avait eu un bras de fer entre l'université de Strasbourg et les autorités parisiennes, universitaires ou CNRS. L'université avait pris le contrôle de la situation, mais avec l'aide du CNRS. Celui-ci reprit ensuite la main, sans faire table rase des spécificités alsaciennes, tandis que la faculté continuait à financer le laboratoire strasbourgeois. L'intégration du CRN à l'IN2P3, centre de statut national, ne changea significativement ni ses orientations ni son fonctionnement. Paris assurait une gestion concentrée des moyens, avec une répartition locale des projets. En matière de politique de la recherche, c'était une sorte de compromis entre le grand laboratoire national, que certains réclamaient, et le soutien aux équipes régionales.

Le CRM, du CNRS à l'université

19 Toute autre est l'histoire de la naissance du Centre de recherches sur les macromolécules. Elle est liée à l'initiative d'un physicien atypique, Charles Sadron, qui travaillait en marge de l'université quoique lui appartenant. Ce projet fut soutenu dès le départ par le CNRS. À son retour de déportation, Charles Sadron n'avait pas l'intention de reprendre un poste à Strasbourg. Il avait pour cela plusieurs raisons. En premier lieu, son centre d'intérêt personnel le poussait vers une recherche originale en France à cette époque, les macromolécules. Ses ambitions dans ce domaine étaient anciennes, et il avait, dès avant la guerre, élaboré un projet d'étude des matières plastiques pour le ministère de la Guerre. Strasbourg n'offrait rien pour un tel développement. En second lieu, il avait conçu un projet d'organisation de la recherche pour la France fondé sur l'idée « d'usines à recherche », inspirées du modèle américain qu'il connaissait bien pour avoir fait un stage post-doc. au CalTec (Californian Technology Institut), à Pasadena, en 1934. Il souhaitait la création d'un laboratoire de taille nationale, qui aurait regroupé les meilleurs spécialistes de la question. Strasbourg lui paraissait une ville trop petite, géographiquement trop isolée pour devenir une capitale scientifique ou le siège d'un grand laboratoire national. Charles Sadron comptait donc sur ses amis Edmond Bauer et Émile Terroine, anciens collègues de Strasbourg en poste ou mission à Paris, pour appuyer sa candidature à Paris. Il essuya une série d'échecs, au Collège de France et à la Sorbonne. Il arrivait trop tard et subissait la concurrence de Georges Champetier bien implanté à Paris et soucieux de préserver son pré carré ; mais surtout l'objet même de ses projets passait mal. Sa définition disciplinaire manquait de clarté pour l'époque. Les macromolécules étaient encore inclassables d'un point de vue académique. Ce qu'il proposait n'était ni de la physique, ni de la chimie, ni de la biologie. Mais pourquoi ne pas les étudier à Strasbourg? On l'y encourageait même, pour développer les grandes équipes de province. La création du centre strasbourgeois fut donc décidée par Paris.

Charles Sadron se vit contraint d'accepter la chaire de physique laissée vacante par le départ de Strasbourg de Louis Néel. Il ne désespéra cependant jamais de trouver un jour le moyen de réaliser ses ambitions : "Je vais aller construire le CRM à Monte Carlo. Le Prince, tout à coup, s'intéresse à la science plutôt qu'à son jardin et son aquarium ", rappelait avec humour Henri Benoît, le citant, lors de la cérémonie du $30^{\mathrm{e}}$ anniversaire $\mathrm{du} \mathrm{CRM}^{10}$. Faisant contre mauvaise fortune bon cœur, Charles Sadron entreprit de légitimer le champ nouveau des macromolécules. Il réunit une équipe de jeunes nouvellement arrivés, tel Henri Benoît, et obtint de la fondation Rockefeller, dont il avait autrefois été boursier, des fonds qui lui permirent d'organiser, dès 1946, un colloque international auquel participèrent ceux qui étaient les plus avancés en la matière, des Américains, des Néerlandais et des Britanniques. Mais la faculté ne voulait 
rien entendre. Il lui fallut négocier au niveau national pour obtenir la création d'un laboratoire propre du CNRS. L'obtention par Charles Sadron en 1946 du prix Holweck, décerné alternativement par l'Institute of Physics britannique à un Français et par la Société française de physique à un Britanique, pesa vraisemblablement dans la balance. Le Centre d'études de la physique des macromolécules (CEPM) fut créé le 7 mai 1947. C'était le premier laboratoire propre du CNRS : « le LP 1 ». Ses statuts, rédigés par Émile Terroine, furent approuvés par Georges Teissier, qui avait remplacé Frédéric Joliot à la direction du CNRS.

Le laboratoire était constitué de deux universitaires, Charles Sadron, professeur, et Henri Benoît, maître de conférences, et de six chargés de recherche du CNRS (Georges Vallet, Roger Cerf, Erwin Heintz, Joseph Leray, Jean-Baptiste Donnet, Gaston Scheibling). Situés dans les caves de l'université, les locaux du laboratoire étaient exigus et peu confortables. Du matériel fut récupéré dans ce que les Allemands avaient laissé derrière eux et dont personne ne s'occupait, dans les instituts délabrés. Le CNRS, l'université et diverses entreprises industrielles participaient au budget. L'originalité du laboratoire tenait à la fois à l'orientation et à l'organisation de la recherche. Formé au magnétisme par Pierre Weiss, Charles Sadron avait abandonné assez tôt ce champ mais en avait conservé le goût des expériences et des mesures. Son passage chez le professeur Von Karman, à Pasadena, lorsqu'il était "boursier Rockefeller ", l'avait plongé dans l'univers des grands laboratoires américains. Il en était revenu impressionné par la variété des statuts des personnels, qu'il s'agisse de chercheurs académiques ou d'ingénieurs, par la taille des équipes, et surtout par le foisonnement des sujets traités. Il avait alors réorienté ses propres recherches sur les grosses molécules et s'était lancé dans des mesures physiques statistiques. Dans le même temps, il s'était mis à travailler en liaison avec des chimistes et des biologistes. Son objectif était d'obtenir la création d'un centre d'études sur les macromolécules composé de trois sections: physicochimie des polymères, polymères biologiques, synthèse des polymères. Les trois disciplines de physique, chimie et biologie devaient collaborer dans des programmes communs, fortement imbriqués les uns aux autres. Il fallait caractériser les substances étudiées, mesurer les molécules, les analyser et établir des relations entre les caractéristiques des molécules et certaines propriétés du matériau. Il s'agissait donc de poursuivre à la fois la modélisation et l'approche expérimentale. Les contacts avec l'industrie se développèrent. Les industriels apportaient leurs échantillons. Comme les calibrages posaient parfois problème, il fallut prévoir des étalonnages et créer les molécules nécessaires à cet effet. Ainsi «par un détour industriel, le groupe était encouragé à développer pour lui-même la maitrise de l'instrumentation et à produire les substances adaptées ${ }^{11} »$. Comme l'a qualifié François Jacq dans sa thèse, le laboratoire de Charles Sadron devint dans un premier temps un centre de production de techniques de mesures. La reconnaissance du laboratoire strasbourgeois fut internationale dès 1946, après le premier congrès tenu à Strasbourg. Cette ouverture sur l'étranger devint et resta une caractéristique du laboratoire et se perpétua par les allers et venues de chercheurs et d'étudiants. L'élargissement des recherches à la chimie et à la biologie se fit grâce au recrutement, dès 1948, de nouveaux chercheurs: Paul Rempp et Madeleine Champagne. Ils s'occupèrent de la synthèse des polymères. Charles Sadron obtint aussi le soutien de biologistes, comme R. Signer à Berne et André Boivin, de la faculté de médecine de Strasbourg. Malheureusement ce dernier mourut en 1949 et ne fut pas remplacé dans l'immédiat. 
22 La question des locaux devint rapidement cruciale. Le Centre ne pouvait se contenter d'une cave! L'université n'en faisait pas une priorité. En fin de compte, un terrain jouxtant l'Institut de mécanique des fluides, mais hors de l'enceinte du campus, fut concédé par la ville. Le Centre de recherches sur les macromolécules put ainsi être créé. Ceci provoqua un retournement de la position de l'université. Le doyen Pierre Lacroute, doué d'une grande capacité d'anticipation, se saisit de l'occasion pour associer officiellement l'université au projet du CNRS. Le fait que la gestion des laboratoires propres relevait des comités de direction des laboratoires, rattachés à la Direction générale et au Directoire, facilita "l'infiltration" de l'université et favorisa ensuite l'association.

Les négociations donnèrent lieu à la signature d'une convention entre le recteur René Hubert et Gaston Dupouy, directeur du CNRS, le 6 mai 1952, à Paris. Il est vraisemblable que, selon sa tradition, le CNRS ait proposé ses services juridiques pour la rédaction de la convention. Le directeur était nommé par le CNRS, mais l'université avait droit à des représentants. Par accord tacite, le directeur était un enseignant de l'université. présence du professeur Hermann Staudinger, prix Nobel de chimie pour sa découverte des macromolécules, et de nombreuses personnalités. Cette manifestation eut aussi pour conséquence qu'Henri Longchambon suggéra la création du CRN sur le modèle du CRM car le principe de la convention se révélait visiblement opératoire.

La convention marquait une étape importante à plusieurs égards. Tout d'abord parce que l'université tenait à être partie prenante dans ce qui constituait avant la lettre une équipe mixte. C'était faire d'ailleurs faire preuve d'intelligence politique. Elle en tirait des avantages dans la mesure où elle participait au choix du directeur, qui fut de fait toujours un universitaire, et à l'organisation du recrutement des enseignants. Sur le plan scientifique, elle bénéficiait de la double signature des publications, ce qui lui assurait une plus grande notoriété. Sur le plan pédagogique, à l'époque de la montée en puissance de l'université de masse, elle autorisait ses étudiants à faire des stages au CRM dans le cadre de leurs diplômes universitaires. Le CRM étant considéré comme l'équivalent d'un laboratoire de l'université, il participait ainsi directement à la formation initiale des futurs chercheurs. On ne peut s'empêcher de voir dans la proposition du doyen Pierre Lacroute les prémisses d'une politique universitaire de la recherche telle qu'on la connut plus tard de manière plus systématique.

Le CNRS de son côté jouait cette fois non pas la carte de l'agence de moyens, mais celle d'un organisme de recherche, en assumant le rôle de maître d'œuvre d'un grand laboratoire. La recherche appliquée était explicitement citée dans les objectifs du CRM, ce qui prolongeait ses rapprochements antérieurs avec l'industrie. Enfin, l'enseignement et la recherche étaient délibérément associés, ce qui devait permettre la constitution d'un vivier de recrutement. Toutefois, le principe même de ce texte pouvait surprendre au regard des prises de position de Charles Sadron, qui défendait publiquement et avec véhémence une recherche coupée de l'Université. Il avait consenti là à une alliance contre-nature.

\section{Du CRM à l'Institut Charles Sadron}

Le CRM se développa dans plusieurs directions et prit de l'ampleur. Les effectifs passèrent de 11 personnes en 1952 ( 7 chercheurs, 4 techniciens) à 30 en 1954 (17 chercheurs, 11 techniciens et 2 étrangers), puis 50 en 1956 (30 chercheurs, 20 techniciens), 125 en 1958 et 200 en 1965. L'absence d'institut de science appliquée se 
faisant sentir, la création d'une école fut mise à l'ordre du jour. Le projet, lancé par Charles Sadron en 1961 auprès de la direction de l'Enseignement supérieur, fut accepté par un arrêté du 19 décembre 1963, après quelques tergiversations de la part du ministère et des concessions de l'université.: «L'École d'application des hauts polymères (EAHP) est un institut de la faculté des sciences de Strasbourg. Elle a pour but de former des ingénieurs spécialisés dans le domaine des hauts polymères. Son siège est à Strasbourg. Elle comprend deux sections, l'une à Mulhouse, l'autre à Strasbourg. $»^{12}$

Une chaire de physicochimie industrielle fut créée pour le directeur C. Wippler, qui avait été chercheur au CNRS et avait également acquis une expérience industrielle chez Saint-Gobain. Un nouveau bâtiment, construit en partie grâce à un financement de Siemens, ouvrit en 1968. Un hall de transformation des matières plastiques fut installé dans les locaux de l'école pour servir de pont entre les recherches théoriques du CRM et les besoins de recherche appliquée de l'industrie. L'École formait des ingénieurs en deux ans, en recrutant soit des ingénieurs sortant des écoles de chimie, soit des étudiants ayant une maîtrise en sciences. Une des difficultés institutionnelles de cette école fut d'obtenir le droit de conférer le statut d'ingénieur aux sortants (bac + 6).

Henri Benoît rappelle toutefois que «le CNRS, à une époque où il était moins sensible aux retombées industrielles que maintenant, regardait avec suspicion le développement de l'École et m'avait demandé d'installer une barrière entre les deux instituts. Cette barrière resta symbolique, car la collaboration était nécessaire aussi bien pour les problèmes d'enseignement que pour les problèmes de recherche ${ }^{13} »$. De fait, le CNRS infléchit à nouveau sa politique dans les années 1980 et chercha à privilégier les rapports avec l'industrie. Dans cette optique, il parut souhaitable d'opérer le regroupement du CRM et de l'EAHP, qui donna naissance en 1985 à l'Institut Charles Sadron.

30 La trajectoire du CRM fut donc symétriquement opposée à celle de l'IRN. Formé au sein de l'université de Strasbourg par le CNRS, le Centre évolua vers une entité associant la recherche, l'enseignement et la formation d'ingénieurs spécialisés. Le choix du CNRS comme organisme de rattachement permit, au départ, le lancement d'une activité originale peu présente dans l'Université française, débouchant sur des contacts avec l'industrie. La convention associa plus étroitement l'université au développement des recherches. La création de l'École favorisa les liens entre la recherche et la formation industrielle. Le CRM joua ainsi un rôle non négligeable, voire même déterminant, à la fois dans l'histoire des disciplines scientifiques en France et dans la construction de solutions institutionnelles dans les relations entre l'Université et le CNRS.

Leçons de ces deux expériences

31 Les débats nationaux sur les missions du CNRS et de l'Université, parfois si orageux dans l'immédiat après-guerre et jusqu'au lendemain du colloque de Caen, portaient sur deux questions fondamentales: les relations entre recherche et enseignement d'une part et entre recherche pure et recherche appliquée d'autre part.

32 En réponse à la première question, cette rapide histoire comparée du CRN et du CRM tend à montrer qu'à Strasbourg on ne voulait pas isoler la recherche de toute forme d'enseignement, que ce soit par besoin de former de futurs chercheurs ou encore par nécessité interne au développement des problématiques scientifiques. Les trajectoires des deux centres ont donc logiquement convergé vers une solution de collaboration institutionnelle. Le CRN étant intégré au grand laboratoire CNRS/IN2P3 et le CRM 
créant une École des hauts polymères, la question des relations entre l'enseignement et la recherche trouva une solution interactive et féconde dans chacune des deux entités.

Dans le domaine des champs disciplinaires, on observe une tentative similaire dans les deux laboratoires d'associer plus étroitement les chimistes et les biologistes aux physiciens, selon leurs besoins spécifiques. Le recrutement des chimistes posa toutefois problème au CRM. S'il se trouvait sur place de nombreux jeunes chimistes, issus de l'École supérieure de chimie de Strasbourg, et qui venaient faire de la physique au CRM, il n'y eut en revanche qu'un seul professeur de chimie, envoyé de Paris par Georges Champetier et ayant eu une carrière sans lien avec les polymères. La chimie organique, après le départ d'Albert Kirrmann, ne considéra jamais la chimie macromoléculaire comme une priorité et ne favorisa pas la nomination d'assistants ou de professeurs dans cette discipline. La collaboration interdisciplinaire ne fut donc pas facile à mettre en œuvre. Du coup, la capacité à évoluer par remodelage des équipes ou réorientation des travaux fut ralentie. Le système se rigidifia quelque peu. L'attitude mandarinale des deux directeurs en fin de carrière eut des effets pervers. Leur volonté de rester directeur en titre alors que leur présence à Strasbourg était de plus en plus épisodique nuisit beaucoup aux équipes. Lorsque Charles Sadron quitta officiellement Strasbourg en 1968, après dix ans d'absence de fait, il tenta même d'entraîner dans son sillage l'équipe de biologistes du CRM, ce qui ébranla l'architecture du Centre. Le développement des capacités scientifiques et organisationnelles de ses successeurs fut freiné.

34 En ce qui concerne la question de la recherche pure opposée à la recherche appliquée, la divergence entre les deux centres strasbourgeois fut nette. Charles Sadron souhaitait des contacts avec l'industrie. Serge Gorodetzky insistait sur l'intérêt de la science pure. On retrouvait là l'opposition fondamentale entre les deux institutions. Et pourtant, au bout du compte, ni l'un ni l'autre de ces modèles ne fut tout à fait opératoire ou satisfaisant. Ainsi par exemple, Charles Sadron ne parvint jamais à mettre en place les projets ambitieux qu'il proposa à plusieurs reprises. Quelles furent les causes de ces échecs? Il faut tout d'abord se pencher sur les projets de Charles Sadron. Comment, dans ses propositions "d'usines de recherche", concevait-il les relations entre les chercheurs et l'industrie, entre les chercheurs eux-mêmes? Ce qui l'intéressait, c'était la dynamique entre les problèmes posés par la théorie et ceux que l'application à la production faisait naître. Un laboratoire d'entreprise devait servir de pont entre les résultats des recherches universitaires et la production industrielle. Pour justifier les collaborations, Charles Sadron avançait des raisons politiques (l'intérêt national), méthodologiques (les instruments, les mesures) et épistémologiques (l'avancement de la science). Or, la demande des entreprises était très ambiguë: mêmes celles qui acceptaient de collaborer ne le faisaient pas toujours pleinement par choix économique. C'étaient souvent des individus «éclairés» qui se lançaient dans l'aventure des processus complets d'innovation, alliant recherche académique, recherche industrielle et développement, comme par exemple Corvazier, directeur technique chez Duco (entreprise de peintures), ou Pierre Piganiol chez Saint-Gobain. Le travail de collaboration visait aussi bien le développement de l'entreprise elle-même que celui de la branche. Les intérêts immédiats de l'entreprise n'étaient pas visés, si bien qu'au premier changement de direction, à la première difficulté de trésorerie, les activités de recherche étaient dénoncées comme des poids morts. François Jacq cite la réaction de la direction de Duco, rendant le service de recherche responsable de ses déboires financiers : «Si les membres de ce laboratoire n'ont pas ce rendement, c'est 
qu'au lieu de travailler pour les ventes, ils ont fait « de la Science » et perdu leur temps à des problèmes scientifiques sans portée pratique ${ }^{14} »$. Madame N. Dubois-Daune, venue de l'Université et recrutée par l'entreprise, fut remerciée. D'autres entreprises qui auraient pu se lancer dans l'aventure ne montrèrent aucune ambition et ne prirent aucun risque. En 1952, Charles Sadron ne réussit pas à convaincre A. Auberger, le président du Port autonome de Strasbourg, de se battre pour obtenir la création d'une raffinerie qui aurait permis de créer une entreprise de caoutchouc synthétique. Le syndicat professionnel du caoutchouc lui-même ne fut guère audacieux dans le domaine, les intérêts de Michelin l'emportant finalement sur toute autre stratégie. Si un très petit laboratoire, sorte d'annexe strasbourgeoise du Laboratoire de recherche et de Contrôle du Caoutchouc, fut installé en 1956 par C. Ortlieb dans un baraquement de la cour du CRM, il fut forcé de fermer en 1958, faute de crédits suffisants de la part de la profession. Son objectif était l'étude des polymères industriels et des élastomères, ce qui aurait constitué un bon exemple de collaboration entre la recherche et l'industrie. Il faut donc insister sur l'attitude du patronat industriel français, la sousindustrialisation de la France et l'arriération des mentalités encore très coupées de l'idée de progrès technique.

Un deuxième élément de réponse à l'échec relatif de Charles Sadron tient au fait que le tissu industriel alsacien, fait de très petites unités économiques, ne favorisait pas la $\mathrm{R} \&$ D. Les conditions locales n'étaient pas favorables à ses projets. Enfin, la culture scientifique n'était pas assez développée en France, du fait du système de formation des ingénieurs. La trop grande coupure entre la science et la production dans les enseignements des écoles d'ingénieurs rendait les deux mondes étanches. D'une façon générale en France, l'ingénieur et le savant ne faisaient pas bon ménage. Quelques tentatives furent menées par Charles Sadron et son équipe pour « investir » l'industrie : Georges Vallet se fit embaucher par Saint-Gobain, $\mathrm{M}^{\mathrm{me}} \mathrm{N}$. Daune-Dubois par Duco, comme nous l'avons déjà dit, mais elles restèrent sans lendemain. Le schéma général de l'enseignement supérieur, qui était implicitement à remettre en cause, resta un sujet tabou. L'EAHP fut cantonnée dans une position marginale dans le panorama des écoles d'ingénieurs. Dans ce contexte, la question de la valorisation des travaux scientifiques eut nécessairement du mal à émerger. Charles Sadron constatait que, lorsqu'un problème se présentait, l'entreprise s'adressait ailleurs qu'à l'Université pour le résoudre et cherchait des licences déjà existantes. Aucune politique audacieuse d'exportation de licences françaises vers l'étranger ne vit le jour. Seul le marché intérieur était visé et fourni gratuitement. Le concept de système d'innovation technologique n'était pas encore répandu.

Conclusion

36 Les affrontements sur la conception de la politique de la recherche qui éclatèrent au colloque de Caen furent révélateurs de l'embarras à concevoir des liens entre la recherche, son financement et ses applications. Charles Sadron fut un des ténors, quoique physiquement absent lors du colloque, de la défense d'une recherche combinant la théorie et l'application, mais ses propositions se révélèrent utopiques ou floues, en l'absence d'une dynamique de l'innovation industrielle en France. La frilosité du patronat français, le peu de goût pour l'acculturation scientifique des ingénieurs et le lobby des grandes écoles ne furent pas dénoncés. Paradoxalement, on insista sur les responsabilités des chercheurs dans le creusement du fossé, dans l'absence de langage commun entre entreprises privées et laboratoires publics. 
Alors qu'au niveau national les conflits étaient encore aigus, le contexte local strasbourgeois, avec sa forte tradition de recherche, permit d'avancer, discrètement mais efficacement, vers des solutions reprises plus tard au niveau national. Le CNRS et l'université de Strasbourg servirent de banc d'essai aux conventions et sans doute de modèle.

\section{NOTES}

1.Archives départementales du Bas-Rhin :W1161, 65 (École d'application des hauts polymères), 67 (IRN, local et matériel), 68 (IRN, constitution et statuts). Archives de l'université Louis Pasteur (conventions). Rapports annuels des doyens. Les archives de C. Sadron entreposées dans une cave de l'Institut ont été détruites par une inondation. Les archives personnelles de C. Sadron, que F. Jacq a utilisées dans sa thèse, semblent aujourd'hui perdues.

2.R. Casel, «La recherche nucléaire à Strasbourg ", mémoire de DEA, université Louis Pasteur, Strasbourg, 1993 ; F. Jacq, « Pratiques scientifiques, formes d'organisation et représentations politiques de la science dans la France de l'après-guerre, La «politique de la science» comme énoncé collectif (1944-1962) », thèse de doctorat de l'École nationale supérieure des Mines de Paris, présentée et soutenue le 13 mai 1996. 3.J.-F. Picard, Elisabeth Pradoura, « La longue marche vers le CNRS (1901-1945) » et Antoine Prost : « Les origines de la politique de la recherche en France (1939-1958) », Cahiers pour l'histoire du CNRS, $\mathrm{n}^{\circ}$ 1, Paris, CNRS ÉDITIONS, 1988. M. Charpentier-Morize, «La contribution des « laboratoires propres » du CNRS à la Recherche chimique en France de 1939 à 1973 ", Cahiers pour l'histoire du CNRS, nº 4, Paris, CNRS ÉDITIONS, 1989. «Un CNRS pour quelles avancées scientifiques? Les sciences exactes. Rapport du colloque sur l'histoire du CNRS », et P. Germain, A. Prost, « Histoire de l'organisation de la recherche au CNRS ", Cahiers pour l'histoire du CNRS, nº 6, Paris, CNRS ÉDITIONS, 1989, p. 105. G. Darmon, « La mise en place des instituts nationaux. La difficile naissance de l'IN2P3», Cahiers pour l'histoire du CNRS, n 10, Paris, CNRS ÉDITIONS, 1990.

4.Entretiens avec Henri Benoît, Georges Monsonégo, Guy Ourisson, Gilbert Weill. 5. «Chanson de l'université de Strasbourg ", poème de Louis Aragon publié sous le pseudonyme de François la Colère, le 5 décembre 1944 dans Forces Jeunes, journal de la Résistance imprimé à Clermont-Ferrand.

6.De l'Université aux camps de Concentration, Témoignages strasbourgeois, Presses universitaires de Strasbourg, 1947 (en particulier : «Buchenwald la grande ville », « Le laboratoire du bloc 50 » par Albert Kirrmann, « Le Bloc 61 », « Auschwitz I Stammlager ", « D'Auschwitz à Grossrosen et à Buchenwald », par M. Klein, «à l'usine de Dora " par C. Sadron.

7.F. Perrin, L'organisation de la recherche scientifique en France, Hermann, 1938.

8.La faculté de médecine de Strasbourg était implantée depuis 1872 au cœur des Hospices civils, bénéficiant d'amphithéâtres, de laboratoires de recherches et d'analyses dans les diverses cliniques, et de l'accès direct aux malades. 
9.A. Prost, « Les réformes du CNRS (1959-1966) », Cahiers pour l'histoire du CNRS, n 9, Paris, CNRS ÉDITIONS, 1990.

10.Discours d'H. Benoît, Plaquette du trentenaire du CRM, 18 mars 1977, Strasbourg. Archives de l'Institut de physique de Strasbourg.

11.F. Jacq, op. cit., p. 262.

12.Archives départementales du Bas-Rhin, W1161, 65.

13.H. Benoît, «Les macromolécules à Strasbourg ", Saisons d'Alsace, nº 106, p. 120, 1989.

14.F. Jacq, op. cit., p. 271.

\section{RÉSUMÉS}

Conventions between Strasbourg University and the CNRS: a Pioneer Experience

This article aims at showing how, just after WWII, the dynamics of two institutes located in Strasbourg converged in pioneer conventions between Strasbourg University and the CNRS. Conventions were signed around 1955, i.e. almost fifteen years before what took place in France. The two laboratories involved in this brief compared history are the CRN (Nuclear research Center), belonging to Strasbourg University and led by Serge Gorodetzky, and the CRM (Macromolecular Research Center), belonging to the CNRS and led by Charles Sadron. At that time both laboratories implemented their research in new fields and new disciplines, and their development, although unsimilar, required new kinds of links between research and teaching and interdisciplinarity and industrial innovation. Therefore new ties were to be established between the two institutions. The same pattern was successfully used in the conventions. Backed by a strong tradition of academic research, Strasbourg can be considered as an experimental place in French research, that gave the keynote to modern research policy.

\section{AUTEUR}

\section{FRANÇOISE OLIVIER-UTARD}

Françoise Olivier-Utard est maître de conférences d'histoire contemporaine à l'Institut de recherches interdisciplinaires sur les sciences et les technologies de l'université Louis Pasteur, Strasbourg I. Elle a publié Politique et archéologie, Histoire de la délégation archéologique française en Afghanistan, 1921-1981, Éditions ERC, 1997. À paraître en 2004 et 2005 : Françoise Olivier-Utard, Histoire de l'université de Strasbourg dans l'entre-deuxguerres ; « La Faculté des sciences de l'université française, 1919-1939 », " Pierre Weiss », « Edmond Bauer », in Josiane Olff-Nathan (dir.), Les Facultés de sciences de Strasbourg dans la tourmente, 1870-1945, Éditions La Nuée Bleue. 